\title{
A Model of Mizar Concepts - Unification
}

\author{
Grzegorz Bancerek ${ }^{1}$ \\ Białystok Technical University \\ Poland \\ The University of Finance and Management \\ Białystok-Ełk, Poland
}

\begin{abstract}
Summary. The aim of this paper is to develop a formal theory of Mizar linguistic concepts following the ideas from [6] and [7]. The theory presented is an abstraction from the existing implementation of the Mizar system and is devoted to the formalization of Mizar expressions. The concepts formalized here are: standarized constructor signature, arity-rich signatures, and the unification of Mizar expressions.
\end{abstract}

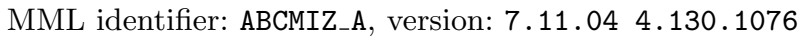

The notation and terminology used in this paper are introduced in the following articles: [20], [21], [12], [22], [10], [14], [13], [17], [18], [15], [1], [8], [11], [2], [3], [4], [19], [16], [5], [9], and [7]. For simplicity the abbreviation $\mathfrak{M}=$ MaxConstrSign is introduced.

\section{Preliminary}

In this paper $i, j$ denote natural numbers.

Next we state two propositions:

(1) For every pair set $x$ holds $x=\left\langle x_{1}, x_{2}\right\rangle$.

(2) For every infinite set $X$ there exist sets $x_{1}, x_{2}$ such that $x_{1}, x_{2} \in X$ and $x_{1} \neq x_{2}$.

In this article we present several logical schemes. The scheme MinimalElement deals with a finite non empty set $\mathcal{A}$ and a binary predicate $\mathcal{P}$, and states that:

\footnotetext{
${ }^{1}$ Partially supported by BTU Grant W/WI/1/06 and UF\&M(B) Teaching Support
} 
There exists a set $x$ such that $x \in \mathcal{A}$ and for every set $y$ such that $y \in \mathcal{A}$ holds not $\mathcal{P}[y, x]$

provided the parameters have the following properties:

- For all sets $x, y$ such that $x, y \in \mathcal{A}$ and $\mathcal{P}[x, y]$ holds not $\mathcal{P}[y, x]$, and

- For all sets $x, y, z$ such that $x, y, z \in \mathcal{A}$ and $\mathcal{P}[x, y]$ and $\mathcal{P}[y, z]$ holds $\mathcal{P}[x, z]$.

The scheme Finite $C$ deals with a finite set $\mathcal{A}$ and a unary predicate $\mathcal{P}$, and states that:

$\mathcal{P}[\mathcal{A}]$

provided the following condition is satisfied:

- For every subset $A$ of $\mathcal{A}$ such that for every set $B$ such that $B \subset A$ holds $\mathcal{P}[B]$ holds $\mathcal{P}[A]$.

The scheme Numeration deals with a finite set $\mathcal{A}$ and a binary predicate $\mathcal{P}$, and states that:

There exists an one-to-one finite sequence $s$ such that $\operatorname{rng} s=\mathcal{A}$ and for all $i, j$ such that $i, j \in \operatorname{dom} s$ and $\mathcal{P}[s(i), s(j)]$ holds $i<j$ provided the parameters satisfy the following conditions:

- For all sets $x, y$ such that $x, y \in \mathcal{A}$ and $\mathcal{P}[x, y]$ holds not $\mathcal{P}[y, x]$, and

- For all sets $x, y, z$ such that $x, y, z \in \mathcal{A}$ and $\mathcal{P}[x, y]$ and $\mathcal{P}[y, z]$ holds $\mathcal{P}[x, z]$.

One can prove the following two propositions:

(3) For every variable $x$ holds $\operatorname{varcl} \operatorname{vars}(x)=\operatorname{vars}(x)$.

(4) Let $\mathfrak{C}$ be an initialized constructor signature and $e$ be an expression of $\mathfrak{C}$. Then $e$ is compound if and only if it is not true that there exists an element $x$ of Vars such that $e=x_{\mathfrak{C}}$.

\section{Standardized Constructor Signature}

Let us note that there exists a quasi-locus sequence which is empty.

Let $\mathfrak{C}$ be a constructor signature. We say that $\mathfrak{C}$ is standardized if and only if the condition (Def. 1) is satisfied.

(Def. 1) Let $o$ be an operation symbol of $\mathfrak{C}$. Suppose $o$ is constructor. Then $o \in$ Constructors and $o_{1}=$ the result sort of $o$ and $\operatorname{Card}\left(\left(o_{2}\right)_{1}\right)=$ len $\operatorname{Arity}(o)$.

The following proposition is true

(5) Let $\mathfrak{C}$ be a constructor signature. Suppose $\mathfrak{C}$ is standardized. Let $o$ be an operation symbol of $\mathfrak{C}$. Then $o$ is constructor if and only if $o \in$ Constructors .

Let us note that $\mathfrak{M}$ is standardized. 
Let us observe that there exists a constructor signature which is initialized, standardized, and strict.

Let $\mathfrak{C}$ be an initialized standardized constructor signature and let $c$ be a constructor operation symbol of $\mathfrak{C}$. The loci of $c$ yielding a quasi-locus sequence is defined by:

(Def. 2) The loci of $c=\left(c_{2}\right)_{\mathbf{1}}$.

Let $\mathfrak{C}$ be a constructor signature. One can verify that there exists a subsignature of $\mathfrak{C}$ which is constructor.

Let $\mathfrak{C}$ be an initialized constructor signature. Note that there exists a constructor subsignature of $\mathfrak{C}$ which is initialized.

Let $\mathfrak{C}$ be a standardized constructor signature. One can verify that every constructor subsignature of $\mathfrak{C}$ is standardized.

One can prove the following two propositions:

(6) Let $S_{1}, S_{2}$ be standardized constructor signatures. Suppose the operation symbols of $S_{1}=$ the operation symbols of $S_{2}$. Then the many sorted signature of $S_{1}=$ the many sorted signature of $S_{2}$.

(7) For every constructor signature $\mathfrak{C}$ holds $\mathfrak{C}$ is standardized iff $\mathfrak{C}$ is a subsignature of $\mathfrak{M}$.

Let $\mathfrak{C}$ be an initialized constructor signature. Observe that there exists a quasi-term of $\mathfrak{C}$ which is non compound.

Let us mention that every element of Vars is pair.

The following propositions are true:

(8) For every element $x$ of Vars such that $\operatorname{vars}(x)$ is natural holds $\operatorname{vars}(x)=0$.

(9) Vars misses Constructors.

(10) For every element $x$ of Vars holds $x \neq *$ and $x \neq$ non.

(11) For every standardized constructor signature $\mathfrak{C}$ holds Vars misses the operation symbols of $\mathfrak{C}$.

(12) Let $\mathfrak{C}$ be an initialized standardized constructor signature and $e$ be an expression of $\mathfrak{C}$. Then

(i) there exists an element $x$ of Vars such that $e=x_{\mathfrak{C}}$ and $e(\emptyset)=\langle x$, term $\rangle$, or

(ii) there exists an operation symbol $o$ of $\mathfrak{C}$ such that $e(\emptyset)=\langle o$, the carrier of $\mathfrak{C}\rangle$ but $o \in$ Constructors or $o=*$ or $o=$ non .

Let $\mathfrak{C}$ be an initialized standardized constructor signature and let $e$ be an expression of $\mathfrak{C}$. Note that $e(\emptyset)$ is pair.

The following propositions are true:

(13) Let $\mathfrak{C}$ be an initialized constructor signature, $e$ be an expression of $\mathfrak{C}$, and $o$ be an operation symbol of $\mathfrak{C}$. Suppose $e(\emptyset)=\langle o$, the carrier of $\mathfrak{C}\rangle$. Then $e$ is an expression of $\mathfrak{C}$ from the result sort of $o$. 
(14) Let $\mathfrak{C}$ be an initialized standardized constructor signature and $e$ be an expression of $\mathfrak{C}$. Then

(i) if $e(\emptyset)_{1}=*$, then $e$ is an expression of $\mathfrak{C}$ from type $\mathbf{C}_{\mathfrak{C}}$, and

(ii) if $e(\emptyset)_{\mathbf{1}}=\mathbf{n o n}$, then $e$ is an expression of $\mathfrak{C}$ from $\mathbf{a d} \mathbf{j}_{\mathfrak{C}}$.

(15) Let $\mathfrak{C}$ be an initialized standardized constructor signature and $e$ be an expression of $\mathfrak{C}$. Then

(i) $\quad e(\emptyset)_{1} \in$ Vars and $e(\emptyset)_{2}=$ term and $e$ is a quasi-term of $\mathfrak{C}$, or

(ii) $e(\emptyset)_{\mathbf{2}}=$ the carrier of $\mathfrak{C}$ but $e(\emptyset)_{1} \in$ Constructors and $e(\emptyset)_{1} \in$ the operation symbols of $\mathfrak{C}$ or $e(\emptyset)_{1}=*$ or $e(\emptyset)_{\mathbf{1}}=$ non .

(16) Let $\mathfrak{C}$ be an initialized standardized constructor signature and $e$ be an expression of $\mathfrak{C}$. If $e(\emptyset)_{1} \in$ Constructors, then $e \in$ (the sorts of Free $\left._{\mathfrak{C}}(\operatorname{Vars} \mathfrak{C})\right)\left(\left(e(\emptyset)_{\mathbf{1}}\right)_{\mathbf{1}}\right)$.

(17) Let $\mathfrak{C}$ be an initialized standardized constructor signature and $e$ be an expression of $\mathfrak{C}$. Then $e(\emptyset)_{\mathbf{1}} \notin$ Vars if and only if $e(\emptyset)_{\mathbf{1}}$ is an operation symbol of $\mathfrak{C}$.

(18) Let $\mathfrak{C}$ be an initialized standardized constructor signature and $e$ be an expression of $\mathfrak{C}$. If $e(\emptyset)_{1} \in$ Vars, then there exists an element $x$ of Vars such that $x=e(\emptyset)_{1}$ and $e=x_{\mathfrak{C}}$.

(19) Let $\mathfrak{C}$ be an initialized standardized constructor signature and $e$ be an expression of $\mathfrak{C}$. Suppose $e(\emptyset)_{1}=*$. Then there exists an expression $\alpha$ of $\mathfrak{C}$ from $\mathbf{a d j}_{\mathfrak{C}}$ and there exists an expression $q$ of $\mathfrak{C}$ from type $\mathbf{e}_{\mathfrak{C}}$ such that $e=\langle *, 3\rangle$-tree $(\alpha, q)$.

(20) Let $\mathfrak{C}$ be an initialized standardized constructor signature and $e$ be an expression of $\mathfrak{C}$. If $e(\emptyset)_{\mathbf{1}}=$ non, then there exists an expression $\alpha$ of $\mathfrak{C}$ from $\mathbf{a d j}_{\mathfrak{C}}$ such that $e=\langle\mathbf{n o n}, 3\rangle$-tree $(\alpha)$.

(21) Let $\mathfrak{C}$ be an initialized standardized constructor signature and $e$ be an expression of $\mathfrak{C}$. Suppose $e(\emptyset)_{\mathbf{1}} \in$ Constructors. Then there exists an operation symbol $o$ of $\mathfrak{C}$ such that $o=e(\emptyset)_{1}$ and the result sort of $o=o_{1}$ and $e$ is an expression of $\mathfrak{C}$ from the result sort of $o$.

(22) Let $\mathfrak{C}$ be an initialized standardized constructor signature and $\tau$ be a quasi-term of $\mathfrak{C}$. Then $\tau$ is compound if and only if $\tau(\emptyset)_{1} \in$ Constructors and $\left(\tau(\emptyset)_{1}\right)_{1}=$ term .

(23) Let $\mathfrak{C}$ be an initialized standardized constructor signature and $\tau$ be an expression of $\mathfrak{C}$. Then $\tau$ is a non compound quasi-term of $\mathfrak{C}$ if and only if $\tau(\emptyset)_{1} \in$ Vars .

(24) Let $\mathfrak{C}$ be an initialized standardized constructor signature and $\tau$ be an expression of $\mathfrak{C}$. Then $\tau$ is a quasi-term of $\mathfrak{C}$ if and only if $\tau(\emptyset)_{1} \in$ Constructors and $\left(\tau(\emptyset)_{1}\right)_{1}=$ term or $\tau(\emptyset)_{1} \in$ Vars .

(25) Let $\mathfrak{C}$ be an initialized standardized constructor signature and $\alpha$ be an expression of $\mathfrak{C}$. Then $\alpha$ is a positive quasi-adjective of $\mathfrak{C}$ if and only if 
$\alpha(\emptyset)_{\mathbf{1}} \in$ Constructors and $\left(\alpha(\emptyset)_{\mathbf{1}}\right)_{\mathbf{1}}=\mathbf{a d j}$.

(26) Let $\mathfrak{C}$ be an initialized standardized constructor signature and $\alpha$ be a quasi-adjective of $\mathfrak{C}$. Then $\alpha$ is negative if and only if $\alpha(\emptyset)_{\mathbf{1}}=$ non .

(27) Let $\mathfrak{C}$ be an initialized standardized constructor signature and $\tau$ be an expression of $\mathfrak{C}$. Then $\tau$ is a pure expression of $\mathfrak{C}$ from type $\mathbf{C}_{\mathfrak{C}}$ if and only if $\tau(\emptyset)_{1} \in$ Constructors and $\left(\tau(\emptyset)_{1}\right)_{1}=$ type .

\section{EXPRESSIONS}

In the sequel $i$ is a natural number, $x$ is a variable, and $\ell$ is a quasi-locus sequence.

An expression is an expression of $\mathfrak{M}$. A valuation is a valuation of $\mathfrak{M}$. A quasiadjective is a quasi-adjective of $\mathfrak{M}$. The subset QuasiAdjs of Free $\mathfrak{M}(\operatorname{Vars} \mathfrak{M})$ is defined as follows:

(Def. 3) QuasiAdjs = QuasiAdjs $\mathfrak{M}$.

A quasi-term is a quasi-term of $\mathfrak{M}$. The subset QuasiTerms of Free $\mathfrak{M}(\operatorname{Vars} \mathfrak{M})$ is defined as follows:

(Def. 4) QuasiTerms = QuasiTerms $\mathfrak{M}$.

A quasi-type is a quasi-type of $\mathfrak{M}$. The functor QuasiTypes is defined as follows:

(Def. 5) QuasiTypes = QuasiTypes $\mathfrak{M}$.

One can verify the following observations:

* QuasiAdjs is non empty,

* QuasiTerms is non empty, and

* QuasiTypes is non empty.

Modes is a non empty subset of Constructors. Then Attrs is a non empty subset of Constructors. Then Funcs is a non empty subset of Constructors.

In the sequel $\mathfrak{C}$ denotes an initialized constructor signature.

The element set-constr of Modes is defined by:

(Def. 6) $\quad$ set-constr $=\langle$ type, $\langle\emptyset, 0\rangle\rangle$.

One can prove the following propositions:

(28) The kind of set-constr $=$ type and the loci of set-constr $=\emptyset$ and the index of set-constr $=0$.

(29) Constructors $=\{$ type, adj, term $\} \times($ QuasiLoci $\times \mathbb{N})$.

(30) $\langle\operatorname{rng} \ell, i\rangle \in \operatorname{Vars}$ and $\ell^{\frown}\langle\langle\operatorname{rng} \ell, i\rangle\rangle$ is a quasi-locus sequence.

(31) There exists $\ell$ such that len $\ell=i$.

(32) For every finite subset $X$ of Vars there exists $\ell$ such that $\operatorname{rng} \ell=\operatorname{varcl} X$.

(33) Let $X, o$ be sets and $p$ be a decorated tree yielding finite sequence. Given $\mathfrak{C}$ such that $X=\bigcup\left(\right.$ the sorts of $\left.\operatorname{Free}_{\mathfrak{C}}(\operatorname{Vars} \mathfrak{C})\right)$. If $o$-tree $(p) \in X$, then $p$ is a finite sequence of elements of $X$. 
Let us consider $\mathfrak{C}$ and let $e$ be an expression of $\mathfrak{C}$. An expression of $\mathfrak{C}$ is called a subexpression of $e$ if:

(Def. 7) $\quad$ It $\in \operatorname{Subtrees}(e)$.

The functor constrs $e$ is defined by:

(Def. 8) constrs $e=\pi_{1}(\operatorname{rng} e) \cap\{o: o$ ranges over constructor operation symbols of $\mathfrak{C}\}$.

The functor main-constr $e$ is defined by:

(Def. 9) main-constr $e=\left\{\begin{array}{l}e(\emptyset)_{\mathbf{1}}, \text { if } e \text { is compound, } \\ \emptyset, \text { otherwise. }\end{array}\right.$

The functor args $e$ yields a finite sequence of elements of Free $\mathfrak{C}(\operatorname{Vars} \mathfrak{C})$ and is defined by:

(Def. 10) $e=e(\emptyset)$-tree $(\operatorname{args} e)$.

Next we state three propositions:

(34) For every $\mathfrak{C}$ holds every expression $e$ of $\mathfrak{C}$ is a subexpression of $e$.

(35) main-constr $\left(x_{\mathfrak{C}}\right)=\emptyset$.

(36) Let $c$ be a constructor operation symbol of $\mathfrak{C}$ and $p$ be a finite sequence of elements of QuasiTerms $\mathfrak{C}$. If $\operatorname{len} p=\operatorname{len} \operatorname{Arity}(c)$, then main-constr $(\vec{c}(p))=c$.

Let us consider $\mathfrak{C}$ and let $e$ be an expression of $\mathfrak{C}$. We say that $e$ is constructor if and only if:

(Def. 11) $e$ is compound and main-constr $e$ is a constructor operation symbol of $\mathfrak{C}$.

Let us consider $\mathfrak{C}$. Observe that every expression of $\mathfrak{C}$ which is constructor is also compound.

Let us consider $\mathfrak{C}$. Observe that there exists an expression of $\mathfrak{C}$ which is constructor.

Let us consider $\mathfrak{C}$ and let $e$ be a constructor expression of $\mathfrak{C}$. One can verify that there exists a subexpression of $e$ which is constructor.

Let $S$ be a non void signature, let $X$ be a non empty yielding many sorted set indexed by $S$, and let $\tau$ be an element of Free $_{S}(X)$. Observe that $\operatorname{rng} \tau$ is relation-like.

One can prove the following proposition

(37) For every constructor expression $e$ of $\mathfrak{C}$ holds main-constr $e \in \operatorname{constrs} e$.

\section{ARITy}

For simplicity, we follow the rules: $\alpha$ is a quasi-adjective, $\tau, \tau_{1}, \tau_{2}$ are quasiterms, $\vartheta$ is a quasi-type, and $c$ is an element of Constructors.

Let $\mathfrak{C}$ be a non void signature. We say that $\mathfrak{C}$ is arity-rich if and only if the condition (Def. 12) is satisfied. 
(Def. 12) Let $n$ be a natural number and $s$ be a sort symbol of $\mathfrak{C}$. Then $\{o$;o ranges over operation symbols of $\mathfrak{C}$ : the result sort of $o=s \wedge \operatorname{len} \operatorname{Arity}(o)=n\}$ is infinite.

Let $o$ be an operation symbol of $\mathfrak{C}$. We say that $o$ is nullary if and only if:

(Def. 13) $\operatorname{Arity}(o)=\emptyset$.

We say that $o$ is unary if and only if:

(Def. 14) len $\operatorname{Arity}(o)=1$.

We say that $o$ is binary if and only if:

(Def. 15) len $\operatorname{Arity}(o)=2$.

The following proposition is true

(38) Let $\mathfrak{C}$ be a non void signature and $o$ be an operation symbol of $\mathfrak{C}$. Then

(i) if $o$ is nullary, then $o$ is not unary,

(ii) if $o$ is nullary, then $o$ is not binary, and

(iii) if $o$ is unary, then $o$ is not binary.

Let $\mathfrak{C}$ be a constructor signature. Observe that non $_{\mathfrak{C}}$ is unary and $*_{\mathfrak{C}}$ is binary.

Let $\mathfrak{C}$ be a constructor signature. Note that every operation symbol of $\mathfrak{C}$ which is nullary is also constructor.

The following proposition is true

(39) Let $\mathfrak{C}$ be a constructor signature. Then $\mathfrak{C}$ is initialized if and only if there exists an operation symbol $m$ of type $\mathbf{C}_{\mathfrak{C}}$ and there exists an operation symbol $\alpha$ of $\mathbf{a d j}_{\mathfrak{C}}$ such that $m$ is nullary and $\alpha$ is nullary.

Let $\mathfrak{C}$ be an initialized constructor signature. One can verify that there exists an operation symbol of type $\mathbf{C}_{\mathfrak{C}}$ which is nullary and constructor and there exists an operation symbol of $\mathbf{a d} \mathbf{j}_{\mathfrak{C}}$ which is nullary and constructor.

Let $\mathfrak{C}$ be an initialized constructor signature. Observe that there exists an operation symbol of $\mathfrak{C}$ which is nullary and constructor.

One can check that every non void signature which is arity-rich has also an operation for each sort and every constructor signature which is arity-rich is also initialized.

One can check that $\mathfrak{M}$ is arity-rich.

Let us mention that there exists a constructor signature which is arity-rich and initialized.

Let $\mathfrak{C}$ be an arity-rich constructor signature and let $s$ be a sort symbol of $\mathfrak{C}$. One can verify the following observations:

* there exists an operation symbol of $s$ which is nullary and constructor,

* there exists an operation symbol of $s$ which is unary and constructor, and

* there exists an operation symbol of $s$ which is binary and constructor. 
Let $\mathfrak{C}$ be an arity-rich constructor signature. One can check that there exists an operation symbol of $\mathfrak{C}$ which is unary and constructor and there exists an operation symbol of $\mathfrak{C}$ which is binary and constructor.

The following proposition is true

(40) Let $o$ be a nullary operation symbol of $\mathfrak{C}$. Then $\langle o$, the carrier of $\mathfrak{C}\rangle$-tree $(\emptyset)$ is an expression of $\mathfrak{C}$ from the result sort of $o$.

Let $\mathfrak{C}$ be an initialized constructor signature and let $m$ be a nullary constructor operation symbol of type $\mathbf{e}_{\mathfrak{C}}$. Then $m_{\mathrm{t}}$ is a pure expression of $\mathfrak{C}$ from type . .

Let $c$ be an element of Constructors. The functor ${ }^{\circledR} c$ yielding a constructor operation symbol of $\mathfrak{M}$ is defined by:

(Def. 16) ${ }^{\circledR} c=c$.

Let $m$ be an element of Modes. Then ${ }^{\circledR} m$ is a constructor operation symbol of type $\mathbf{M}_{\mathfrak{M}}$.

Let us note that ${ }^{\circledR}$ set-constr is nullary.

We now state the proposition

(41) Arity $\left({ }^{@}\right.$ set-constr $)=\emptyset$.

The quasi-type set-type is defined by:

(Def. 17) $\quad$ set-type $=\emptyset_{\text {QuasiAdjs } \mathfrak{M}} *\left({ }^{@} \text { set-constr }\right)_{\mathrm{t}}$.

The following proposition is true

(42) adjs set-type $=\emptyset$ and the base of set-type $=\left({ }^{@} \text { set-constr }\right)_{\mathrm{t}}$.

Let $\ell$ be a finite sequence of elements of Vars. The functor $\operatorname{args} \ell$ yields a finite sequence of elements of QuasiTerms $\mathfrak{M}$ and is defined as follows:

(Def. 18) $\operatorname{len} \operatorname{args} \ell=\operatorname{len} \ell$ and for every $i$ such that $i \in \operatorname{dom} \ell$ holds $(\operatorname{args} \ell)(i)=$ $\left(\ell_{i}\right)_{\mathfrak{M}}$.

Let us consider $c$. The base expression of $c$ yields an expression and is defined as follows:

(Def. 19) The base expression of $c=\left({ }^{@} c\right) \rightarrow(\operatorname{args}($ the loci of $c))$.

Next we state several propositions:

(43) For every operation symbol $o$ of $\mathfrak{M}$ holds $o$ is constructor iff $o \in$ Constructors .

(44) For every nullary operation symbol $m$ of $\mathfrak{M}$ holds main-constr $\left(m_{\mathrm{t}}\right)=m$.

(45) For every unary constructor operation symbol $m$ of $\mathfrak{M}$ and for every $\tau$ holds main-constr $(m(\tau))=m$.

(46) For every $\alpha$ holds main-constr $\left(\right.$ non $\left._{\mathfrak{M}}(\alpha)\right)=$ non .

(47) For every binary constructor operation symbol $m$ of $\mathfrak{M}$ and for all $\tau_{1}, \tau_{2}$ holds main-constr $\left(m\left(\tau_{1}, \tau_{2}\right)\right)=m$.

(48) For every expression $q$ of $\mathfrak{M}$ from type $_{\mathfrak{M}}$ and for every $\alpha$ holds main-constr $(* \mathfrak{M}(\alpha, q))=*$. 
Let $\vartheta$ be a quasi-type. The functor constrs $\vartheta$ is defined by:

(Def. 20) $\operatorname{constrs} \vartheta=\operatorname{constrs}($ the base of $\vartheta) \cup \bigcup\{\operatorname{constrs} \alpha: \alpha \in \operatorname{adjs} \vartheta\}$.

The following two propositions are true:

(49) For every pure expression $q$ of $\mathfrak{M}$ from type $_{\mathfrak{M}}$ and for every finite subset $A$ of QuasiAdjs $\mathfrak{M}$ holds constrs $(A * q)=$ constrs $q \cup \bigcup\{\operatorname{constrs} \alpha: \alpha \in A\}$.

(50) $\operatorname{constrs}(\alpha * \vartheta)=\operatorname{constrs} \alpha \cup \operatorname{constrs} \vartheta$.

\section{UNIFICATION}

Let $\mathfrak{C}$ be an initialized constructor signature and let $\tau, p$ be expressions of $\mathfrak{C}$. We say that $\tau$ matches $p$ if and only if:

(Def. 21) There exists a valuation $f$ of $\mathfrak{C}$ such that $\tau=p[f]$.

Let us note that the predicate $\tau$ matches $p$ is reflexive.

The following proposition is true

(51) For all expressions $\tau_{1}, \tau_{2}, \tau_{3}$ of $\mathfrak{C}$ such that $\tau_{1}$ matches $\tau_{2}$ and $\tau_{2}$ matches $\tau_{3}$ holds $\tau_{1}$ matches $\tau_{3}$.

Let $\mathfrak{C}$ be an initialized constructor signature and let $A, B$ be subsets of QuasiAdjs $\mathfrak{C}$. We say that $A$ matches $B$ if and only if:

(Def. 22) There exists a valuation $f$ of $\mathfrak{C}$ such that $B[f] \subseteq A$.

Let us note that the predicate $A$ matches $B$ is reflexive.

The following proposition is true

(52) For all subsets $A_{1}, A_{2}, A_{3}$ of QuasiAdjs $\mathfrak{C}$ such that $A_{1}$ matches $A_{2}$ and $A_{2}$ matches $A_{3}$ holds $A_{1}$ matches $A_{3}$.

Let $\mathfrak{C}$ be an initialized constructor signature and let $\vartheta, P$ be quasi-types of

$\mathfrak{C}$. We say that $\vartheta$ matches $P$ if and only if:

(Def. 23) There exists a valuation $f$ of $\mathfrak{C}$ such that (adjs $P)[f] \subseteq$ adjs $\vartheta$ and (the base of $P)[f]=$ the base of $\vartheta$.

Let us note that the predicate $\vartheta$ matches $P$ is reflexive.

One can prove the following proposition

(53) For all quasi-types $\vartheta_{1}, \vartheta_{2}, \vartheta_{3}$ of $\mathfrak{C}$ such that $\vartheta_{1}$ matches $\vartheta_{2}$ and $\vartheta_{2}$ matches $\vartheta_{3}$ holds $\vartheta_{1}$ matches $\vartheta_{3}$.

Let $\mathfrak{C}$ be an initialized constructor signature, let $\tau_{1}, \tau_{2}$ be expressions of $\mathfrak{C}$, and let $f$ be a valuation of $\mathfrak{C}$. We say that $f$ unifies $\tau_{1}$ with $\tau_{2}$ if and only if:

(Def. 24) $\tau_{1}[f]=\tau_{2}[f]$.

The following proposition is true

(54) Let $\tau_{1}, \tau_{2}$ be expressions of $\mathfrak{C}$ and $f$ be a valuation of $\mathfrak{C}$. If $f$ unifies $\tau_{1}$ with $\tau_{2}$, then $f$ unifies $\tau_{2}$ with $\tau_{1}$.

Let $\mathfrak{C}$ be an initialized constructor signature and let $\tau_{1}, \tau_{2}$ be expressions of $\mathfrak{C}$. We say that $\tau_{1}$ and $\tau_{2}$ are unifiable if and only if: 
(Def. 25) There exists a valuation $f$ of $\mathfrak{C}$ such that $f$ unifies $\tau_{1}$ with $\tau_{2}$.

Let us notice that the predicate $\tau_{1}$ and $\tau_{2}$ are unifiable is reflexive and symmetric.

Let $\mathfrak{C}$ be an initialized constructor signature and let $\tau_{1}, \tau_{2}$ be expressions of

$\mathfrak{C}$. We say that $\tau_{1}$ and $\tau_{2}$ are weakly-unifiable if and only if:

(Def. 26) There exists an irrelevant one-to-one valuation $g$ of $\mathfrak{C}$ such that $\operatorname{Var} \tau_{2} \subseteq$ dom $g$ and $\tau_{1}$ and $\tau_{2}[g]$ are unifiable.

Let us note that the predicate $\tau_{1}$ and $\tau_{2}$ are weakly-unifiable is reflexive.

We now state the proposition

(55) For all expressions $\tau_{1}, \tau_{2}$ of $\mathfrak{C}$ such that $\tau_{1}$ and $\tau_{2}$ are unifiable holds $\tau_{1}$ and $\tau_{2}$ are weakly-unifiable.

Let $\mathfrak{C}$ be an initialized constructor signature and let $\tau, \tau_{1}, \tau_{2}$ be expressions of $\mathfrak{C}$. We say that $\tau$ is a unification of $\tau_{1}$ and $\tau_{2}$ if and only if:

(Def. 27) There exists a valuation $f$ of $\mathfrak{C}$ such that $f$ unifies $\tau_{1}$ with $\tau_{2}$ and $\tau=$ $\tau_{1}[f]$.

We now state two propositions:

(56) For all expressions $\tau_{1}, \tau_{2}, \tau$ of $\mathfrak{C}$ such that $\tau$ is a unification of $\tau_{1}$ and $\tau_{2}$ holds $\tau$ is a unification of $\tau_{2}$ and $\tau_{1}$.

(57) For all expressions $\tau_{1}, \tau_{2}, \tau$ of $\mathfrak{C}$ such that $\tau$ is a unification of $\tau_{1}$ and $\tau_{2}$ holds $\tau$ matches $\tau_{1}$ and $\tau$ matches $\tau_{2}$.

Let $\mathfrak{C}$ be an initialized constructor signature and let $\tau, \tau_{1}, \tau_{2}$ be expressions of $\mathfrak{C}$. We say that $\tau$ is a general-unification of $\tau_{1}$ and $\tau_{2}$ if and only if the conditions (Def. 28) are satisfied.

(Def. 28)(i) $\quad \tau$ is a unification of $\tau_{1}$ and $\tau_{2}$, and

(ii) for every expression $u$ of $\mathfrak{C}$ such that $u$ is a unification of $\tau_{1}$ and $\tau_{2}$ holds $u$ matches $\tau$.

\section{Type Distribution}

The following three propositions are true:

(58) Let $n$ be a natural number and $s$ be a sort symbol of $\mathfrak{M}$. Then there exists a constructor operation symbol $m$ of $s$ such that len $\operatorname{Arity}(m)=n$.

(59) Let given $\ell, s$ be a sort symbol of $\mathfrak{M}$, and $m$ be a constructor operation symbol of $s$. If len $\operatorname{Arity}(m)=$ len $\ell$, then $\operatorname{Var}(m(\operatorname{args} \ell))=\operatorname{rng} \ell$.

(60) Let $X$ be a finite subset of Vars. Suppose $\operatorname{varcl} X=X$. Let $s$ be a sort symbol of $\mathfrak{M}$. Then there exists a constructor operation symbol $m$ of $s$ and there exists a finite sequence $p$ of elements of QuasiTerms $\mathfrak{M}$ such that len $p=\operatorname{len} \operatorname{Arity}(m)$ and $\operatorname{vars}(m \rightarrow(p))=X$.

Let $d$ be a partial function from Vars to QuasiTypes. We say that $d$ is even if and only if: 
(Def. 29) For all $x, \vartheta$ such that $x \in \operatorname{dom} d$ and $\vartheta=d(x) \operatorname{holds} \operatorname{vars}(\vartheta)=\operatorname{vars}(x)$.

Let $\ell$ be a quasi-locus sequence. A partial function from Vars to QuasiTypes is said to be a type-distribution for $\ell$ if:

(Def. 30) dom it $=\operatorname{rng} \ell$ and it is even.

We now state the proposition

(61) For every empty quasi-locus sequence $\ell$ holds $\emptyset$ is a type-distribution for $\ell$.

\section{REFERENCES}

[1] Grzegorz Bancerek. König's theorem. Formalized Mathematics, 1(3):589-593, 1990.

[2] Grzegorz Bancerek. Cartesian product of functions. Formalized Mathematics, 2(4):547$552,1991$.

[3] Grzegorz Bancerek. Joining of decorated trees. Formalized Mathematics, 4(1):77-82, 1993.

[4] Grzegorz Bancerek. Subtrees. Formalized Mathematics, 5(2):185-190, 1996.

[5] Grzegorz Bancerek. Institution of many sorted algebras. Part I: Signature reduct of an algebra. Formalized Mathematics, 6(2):279-287, 1997.

[6] Grzegorz Bancerek. On the structure of Mizar types. In Herman Geuvers and Fairouz Kamareddine, editors, Electronic Notes in Theoretical Computer Science, volume 85. Elsevier, 2003.

[7] Grzegorz Bancerek. Towards the construction of a model of Mizar concepts. Formalized Mathematics, 16(2):207-230, 2008, doi:10.2478/v10037-008-0027-x.

[8] Grzegorz Bancerek and Krzysztof Hryniewiecki. Segments of natural numbers and finite sequences. Formalized Mathematics, 1(1):107-114, 1990.

[9] Grzegorz Bancerek and Artur Korniłowicz. Yet another construction of free algebra. Formalized Mathematics, 9(4):779-785, 2001.

[10] Grzegorz Bancerek and Yatsuka Nakamura. Full adder circuit. Part I. Formalized Mathematics, 5(3):367-380, 1996.

[11] Czesław Byliński. Finite sequences and tuples of elements of a non-empty sets. Formalized Mathematics, 1(3):529-536, 1990.

[12] Czesław Byliński. Functions and their basic properties. Formalized Mathematics, 1(1):5565, 1990.

[13] Czesław Byliński. Functions from a set to a set. Formalized Mathematics, 1(1):153-164, 1990

[14] Czesław Byliński. Partial functions. Formalized Mathematics, 1(2):357-367, 1990.

[15] Agata Darmochwał. Finite sets. Formalized Mathematics, 1(1):165-167, 1990.

[16] Beata Perkowska. Free many sorted universal algebra. Formalized Mathematics, 5(1):6774, 1996.

[17] Andrzej Trybulec. Binary operations applied to functions. Formalized Mathematics, 1(2):329-334, 1990.

[18] Andrzej Trybulec. Tuples, projections and Cartesian products. Formalized Mathematics, 1(1):97-105, 1990.

[19] Andrzej Trybulec. Many sorted algebras. Formalized Mathematics, 5(1):37-42, 1996.

[20] Zinaida Trybulec. Properties of subsets. Formalized Mathematics, 1(1):67-71, 1990.

[21] Edmund Woronowicz. Relations and their basic properties. Formalized Mathematics, 1(1):73-83, 1990.

[22] Edmund Woronowicz. Relations defined on sets. Formalized Mathematics, 1(1):181-186, 1990. 